\title{
Exome sequencing identifies frequent mutation of the SWI/SNF complex gene PBRM1 in renal carcinoma
}

\author{
Ignacio Varela ${ }^{1}$, Patrick Tarpey ${ }^{1}$, Keiran Raine ${ }^{1}$, Dachuan Huang ${ }^{2}$, Choon Kiat Ong ${ }^{2}$, Philip \\ Stephens ${ }^{1}$, Helen Davies ${ }^{1}$, David Jones ${ }^{1}$, Meng-Lay Lin ${ }^{1}$, Jon Teague ${ }^{1}$, Graham Bignell ${ }^{1}$, \\ Adam Butler $^{1}$, Juok Cho ${ }^{1}$, Gillian L. Dalgliesh ${ }^{1}$, Danushka Galappaththige ${ }^{1}$, Chris \\ Greenman $^{1}$, Claire Hardy ${ }^{1}$, Mingming Jia ${ }^{1}$, Calli Latimer ${ }^{1}$, King Wai Lau ${ }^{1}$, John Marshall ${ }^{1}$, \\ Stuart McLaren ${ }^{1}$, Andrew Menzies ${ }^{1}$, Laura Mudie ${ }^{1}$, Lucy Stebbings ${ }^{1}$, David A. \\ Largaespada $^{3}$, L.F.A. Wessels ${ }^{4}$, Stephane Richard ${ }^{5,6}$, Richard J Kahnoski ${ }^{7}$, John Anema ${ }^{7}$, \\ David A. Tuveson ${ }^{8}$, Pedro A. Perez-Mancera ${ }^{8}$, Ville Mustonen ${ }^{8}$, Andrej Fischer ${ }^{9,10}$, David J. \\ Adams $^{11}$, Alistair Rust ${ }^{11}$, Waraporn Chan-on ${ }^{2}$, Chutima Subimerb ${ }^{2}$, Karl Dykema ${ }^{12}$, Kyle \\ Furge $^{12}$, Peter J. Campbell ${ }^{1}$, Bin Tean Teh ${ }^{2,14}$, Michael R. Stratton ${ }^{1,15}$, and P. Andrew \\ Futreal $^{1}$
}

${ }^{1}$ Cancer Genome Project, Wellcome Trust Sanger Institute, Hinxton CB10 1SA, UK ${ }^{2}$ NCCS-VARI Translational Research Laboratory, National Cancer Centre Singapore, 11 Hospital Drive, 169610, Singapore ${ }^{3}$ Masonic Cancer Center, University of Minnesota, Minneapolis, MN 55455, USA ${ }^{4}$ Bioinformatics and Statistics, Department of Molecular Biology, The Netherlands Cancer Institute. Plesmanlaan 121, 1066 CX Amsterdam, The Netherlands ${ }^{5}$ Génétique Oncologique EPHE-INSERM U753, Faculté de Médecine Paris-Sud and Institut de Cancérologie Gustave Roussy, 94805 Villejuif ${ }^{6}$ Centre Expert National Cancer Rares INCa "PREDIR", Service d'Urologie, Hôpital de Bicêtre, AP-HP, 94276 Le Kremlin- Bicêtre, France ${ }^{7}$ Department of Urology, Spectrum Health Hospital, Grand Rapids, Michigan 49503, USA. ${ }^{8} \mathrm{Li}$ Ka Shing Centre, Cambridge Research Institute, Cancer Research UK, Robinson Way, Cambridge CB2 ORE, UK ${ }^{9}$ Bioinformatics, Wellcome Trust Sanger Institute, Hinxton CB10 1SA, UK ${ }^{10}$ Institut für Theoretische Physik, Universität zu Köln, Zülpicherstr. 77,50937 Köln, Germany ${ }^{11}$ Experimental Cancer Genetics, Wellcome Trust Sanger Institute, Hinxton CB10 1SA, UK ${ }^{12}$ Laboratory of Computational Biology, Van Andel Research Institute, Grand Rapids, Michigan 49503, USA. 13 Laboratory of Cancer Genetics, Van Andel Research Institute, Grand Rapids, Michigan 49503, USA. ${ }^{14}$ Laboratory of Cancer Therapeutics, DUKE-NUS Graduate Medical School, Singapore ${ }^{15}$ Institute of Cancer Research, Sutton, Surrey SM2 5NG, UK

\section{Summary}

Correspondence and requests for materials should be addressed to BTT (Bin.Teh@vai.org), MRS (mrs@sanger.ac.uk) or PAF (paf@ sanger.ac.uk)..

Author contribution

IV and PT performed the main analytical aspects of the study. PS, HD, GLD, M-LL, GB, CH, LM, SM performed the follow-up sequencing and analyses. KR, DJ, JT, AB, CG, DG, MJ, CL, JM, AM, LS contributed to the data processing, mapping and variant calling informatics. CG and KWL performed statistical analyses. SR, RJK, JA contributed samples and data for the clinical series. DJA, AR, DAL, LFAW, DAT, PAP-M performed the transposon screening and analyses. DH, CKO, WC, CS performed the siRNA and functional work. VM, AF performed the missense mutation analysis. KD, KF and JC performed the expression analyses. PJC, BTT, MRS, PAF directed the study and wrote the manuscript, which all authors have approved.

Author information

Exome sequence data have been deposited at the European Genome-Phenome Archive (http://www.ebi.ac.uk/ega/) hosted by the European Bioinformatics Institute under accession EGAS00001000006 and expression data has been deposited with Gene Expresson Omnibus (http://www.ncbi.nlm.nih.gov/geo/) under accession GEO22316.

The authors declare no competing financial interests. 
The genetics of renal cancer is dominated by inactivation of the $V H L$ tumour suppressor gene in clear cell carcinoma (ccRCC), the commonest histological subtype. A recent large-scale screen of $\sim 3500$ genes by PCR-based exon re-sequencing identified several new cancer genes in ccRCC including $U T X(K D M 6 A)^{1}, J A R I D 1 C(K D M 5 C)$ and SETD2 2 . These genes encode enzymes that demethylate (UTX, JARID1C) or methylate (SETD2) key lysine residues of histone $\mathrm{H} 3$.

Modification of the methylation state of these lysine residues of histone $\mathrm{H} 3$ regulates chromatin structure and is implicated in transcriptional control ${ }^{3}$. However, together these mutations are present in fewer than $15 \%$ of ccRCC, suggesting the existence of additional, currently unidentified cancer genes. Here, we have sequenced the protein coding exome in a series of primary ccRCC and report the identification of the SWI/SNF chromatin remodeling complex gene $P B R M 1^{4}$ as a second major ccRCC cancer gene, with truncating mutations in $41 \%(92 / 227)$ of cases. These data further elucidate the somatic genetic architecture of ccRCC and emphasize the marked contribution of aberrant chromatin biology.

Exome sequencing based on a solution phase capture approach ${ }^{5}$ was performed on seven cases of ccRCC, three of which carry VHL mutations, and matching normal DNAs (See Supplementary information and Supplementary Table 1). Captured material was sequenced using 76 basepair paired-end reads on the Illumina GAIIx platform. After read alignment, variant calling was performed using a naïve Bayesian classifier algorithm for substitutions and a split-read mapping approach ( $\operatorname{PinDel}^{6}$ with substantial cancer-aware output filtering) for insertion/deletions (See Supplementary Material for details). These algorithms aim to identify somatically acquired coding and splice-site variants (i.e. present in the tumour but not in the matching normal), and all mutations reported here were confirmed by PCR-based capillary sequencing. 156 somatic mutations were identified, of which 92 were missense, 9 nonsense, 1 canonical splice site, 1 stop codon read-through, 11 frameshift and 42 synonymous (Supplementary Table 2).

In four cases truncating mutations were indentified in PBRM1. PBRM1 maps to chromosome 3 p21 and encodes the BAF180 protein, the chromatin targeting subunit of the PBAF SWI/SNF chromatin remodelling complex ${ }^{7}$. The gene is comprised of 6 bromodomains involved in binding acetylated lysine residues on histone tails, 2 bromoadjacent homology domains important in protein-protein interaction and an HMG DNA binding domain ${ }^{4}$. PBAF complex-mediated chromatin remodelling is implicated in replication, transcription, DNA repair and control of cell proliferation/differentiation ${ }^{4},{ }^{7}$. The $S M A R C B 1$ and BRG1 components of this complex have inactivating mutations in rhabdoid tumours ${ }^{8}, 9$ and $B R G 1$ mutations have been reported in multiple tumour types ${ }^{10}$. The $P B R M 1$ mutations included three frame-shifting insertions and a nonsense mutation; all judged to be homozygous from SNP array and mutant allele read count data. PBRM1 was not included in our previous PCR-based sequencing screen ${ }^{2}$ and was the only gene, apart from $V H L$, with recurrent truncating mutations in the seven cases screened.

We next sequenced PBRM1 in a further 257 RCC cases, including 36 cases of papillary, chromophobe and other non-ccRCC cancers. Truncating mutations were identified in a remarkable 88/257 (34\%) (Figure 1) of cases, all diagnosed as ccRCC (for full data see Supplementary Tables 3,4). PBRM1 mutations were all found in the context of chromosome $3 p$ loss of heterozygosity (38/38) where SNP array data was available (http:// www.sanger.ac.uk/cgi-bin/genetics/CGP/cghviewer/CghHome.cgi). Two in-frame deletion mutations were identified - a predicted 6 amino-acid deletion (p.M1209_E1214delMFYKKE) in the second BAH (bromo-adjacent homology) domain likely involved in protein-protein interactions within the SWI/SNF complex ${ }^{4}$ and deletion of an isoleucine codon (Ile57) in the first bromodomain (Figure 1). Both deletions remove amino acids conserved to $\mathrm{C}$ elegans and both were in cases with $3 \mathrm{p} \mathrm{LOH}$. The ratio of nine 
missense to zero silent mutations suggests that a proportion of the missense mutations are likely to be pathogenic. Six of nine missense mutations occur in bromodomains and one in the second BAH domain (Figure 1). The bromodomains of PBRM1 have been shown to have preferential binding to different acetylated lysine configurations of histone tails, suggesting they may contribute to "reading" of the histone code ${ }^{11}$. The likelihood of the missense mutations having functional impact was assessed using a scoring system calibrated with protein domain alignments from Pfam (see Supplementary Methods). Three missense mutations (p.T232P, p.A597D and p.H1204P) could be scored with these alignments. This set of mutations was predicted to be deleterious, having a significantly lower mean score than a typical null set of in silico generated random missense mutations falling onto the scorable parts of the gene (p-value 0.01 Figure 2), making these mutations interesting candidates for functional studies.

Four $P B R M 1$ truncating mutations have been previously described in breast cancer ${ }^{12}$. Although there is frequent $3 \mathrm{p} 21 \mathrm{LOH}$ in small-cell lung cancer, no evidence for PBRM1 inactivation was found ${ }^{13}$. To further evaluate the contribution of PBRM1 mutation in human cancer, copy number was evaluated and the coding exons were sequenced through a series of 727 cancer cell lines of various histologies. SNP array copy number analysis (http:// www.sanger.ac.uk/cgi-bin/genetics/CGP/cghviewer/CghHome.cgi) identified one homozygous deletion in the HCC-1143 breast cancer cell line, previously described ${ }^{12}$. Sequencing analysis identified five homozygous truncating mutations (Supplementary Table 5). Frame-shifting deletions were identified in the $V H L$-mutant A704 renal cancer, NCIH2196 small-cell lung cancer and TGBC24TKB gall bladder cancer lines. Nonsense mutations were identified in the NCI-H226 squamous-cell lung cancer and PANC-10-05 pancreatic adenocarcinoma lines. Interestingly, a PBRM1 truncating mutation has been reported in a comprehensive pancreatic cancer mutational screen ${ }^{14}$.

To obtain further support that PBRM1 can act as a cancer gene, we examined data from several insertional mutagenesis screens in mice. Analyses of transposon insertion sites from a forward genetic screen performed using a conditional Sleeping Beauty transposon system ${ }^{15}$ in a mouse pancreatic cancer mode ${ }^{16}$ revealed a significant enrichment of insertion events in Pbrm1 amongst all genes hit using Monte Carlo simulation analyses as previously described ${ }^{17}$. Insertions were found in pancreatic dysplasia, intraductal (panIN) and high grade invasive tumours suggesting Pbrm1 inactivation is an early event in this model. The mixed forward and reverse pattern of insertions is indicative of inactivation, as demonstrated by RT-PCR showing premature termination of the Pbrm 1 cDNA via splicing into the inserted transposon (Figure 3). These data suggest that loss of Pbrm 1 cooperates with Kras in driving pancreatic tumour development in this model. Intriguingly, Setd2, previously implicated human ccRCC, was also found to rank significantly in frequency among all insertion sites and two tumours had both Setd 2 and Pbrm 1 insertions. These comparative oncogenomic data provide independent support for $P B R M 1$ as a cancer gene and suggest further investigation of the role of PBRM1 (and SETD2) in human pancreatic cancer is warranted.

Abrogation of $P B R M 1$ expression via siRNA knockdown in ccRCC cell lines was investigated to assess possible consequences of PBRM1 loss. Greater than $60 \%$ knockdown of $P B R M 1$ RNA and protein resulted in a significant increase in proliferation 4/5 RCC lines (Figure 4A, B and Supplemental Information). No effect was seen, however, in A704 which carries a homozygous truncating $P B R M 1$ mutation and expresses no PBRM1, confirming the specificity of the assay. Further, knockdown of $P B R M 1$ resulted in significantly increased colony formation in soft-agar and increased cell migration (Figure 4C, D), indicative of an increase in transformed phenotype. Taken together, these data support PBRM1 having a tumour suppressor role in ccRCC. 
Transcriptional profiling before and after $P B R M 1$ knockdown was performed using gene expression microarrays. Gene set enrichment analysis following PBRM1 knockdown showed that PBRM1 activity regulates pathways associated with chromosomal instability and cellular proliferation (Figure 4E, Supplementary Table 6), the latter being consistent with previous studies identifying PBRM1 as critical transcriptional regulator of p21/ CDKN1A in breast cancer cell lines ${ }^{12}$ and work showing that PBRM1/Baf180 is implicated in regulating TP53 mediated replicative senescence ${ }^{18}$. The PBAF complex has been shown to localise at kinetochores during mitosis ${ }^{19}$ and SMARCB1 has been implicated in spindle checkpoint $\operatorname{control}^{20}$, which would support the loss of PBRM1 giving rise to a chromosomal instability/spindle checkpoint expression phenotype. It may be of interest to further explore spindle checkpoint control in PBRM1 mutated ccRCC as a potential therapeutic opportunity.

Previous work has demonstrated that VHL loss alone is insufficient for ccRCC tumourigenesis arguing the need for additional genetic events ${ }^{21},{ }^{22}$ (Teh, unpublished) and has further suggested the existence of a 3p21 "gatekeeper" ccRCC mutation based on LOH studies $^{23}$. The data presented here strongly suggest that inactivation of $P B R M 1$ comprises this second major mutation in ccRCC development. Nearly all (36/38) PBRM1 mutant cases fall into the hypoxia signature group as described previously ${ }^{2}$, including $13 / 14$ cases without demonstrable $V H L$ point mutations where expression data is available - further indicating the importance of PBRM1 in typical ccRCC development. The SWI/SNF complex has been implicated in the normal cellular response to hypoxia, with impairment of the complex rendering cells resistant to hypoxia-induced cell cycle arrest ${ }^{24}$, which would be consistent with selection for frequent loss of $P B R M 1$ in ccRCC. Multiple cancers have apparently concomitant $V H L, P B R M 1$ and SETD2 mutations, with all three genes mapping to chromosome $3 \mathrm{p}$, suggesting that the mutations are non-redundant functionally. Half $(55 / 107)$ of cases in this series with a demonstrable VHL mutation ${ }^{2}$ have a PBRM1 mutation. Strikingly, all 9 cases with a SETD2 mutation have a mutation in either PBRM1 or $V H L$, with 6 of 9 cases having mutations in all three genes. Physical linkage of these three ccRCC cancer genes together with their potential interaction may be the key driver for the large scale $3 \mathrm{p} \mathrm{LOH}$ seen in most cases of ccRCC - being particularly parsimonious in requiring only four genetic events to unmask three tumour suppressor genes as opposed to six if the genes were on different chromosomes.

Several other mutated genes of potential interest were identified. In particular, ARIDIA encoding the BAF250A subunit of the SWI/SNF complex was found to have two heterozygous missense mutations - p.R1020K,c.3059G >A and p.L1872P,c.5615T >C. Both cases (PD2126, PD2127) have a PBRM1 truncating mutation. Two homozygous ARID1A deletions were found in SNP 6.0 data (http://www.sanger.ac.uk/cgi-bin/genetics/CGP/conan/ search.cgi) in the LB1047-RCC ccRCC and NCI-SNU-5 gastric carcinoma cell lines and loss of ARID1A expression has been reported in $\mathrm{RCC}^{25}$. Frequent truncating ARID1A truncating mutations have recently been reported in clear cell ovarian carcinoma ${ }^{26,27}$. These data all point to $A R I D 1 A$ being a cancer gene, likely operative in ccRCC. PD2127 was also found to have a heterozygous truncating mutation in $A R I D 5 B$, related to ARID1A and recently implicated in childhood acute lymphoblastic leukaemia susceptibility ${ }^{28}$. The extent to which the other mutated genes identified here contribute to ccRCC will await large-scale follow-up screens. Similarly, exome and whole genome sequencing on a large number of cases is likely to yield further insights.

The identification of a second major cancer gene in ccRCC further defines the genetic and molecular architecture of this tumour type. It is remarkable that $P B R M 1$, like the majority of the other non-VHL mutated cancer genes identified in ccRCC, is involved in chromatin regulation - again at least in part at the level of histone $\mathrm{H} 3$ modification and recognition. Understanding the contribution of PBRM1 mutation to clinical disease progression and 
outcome as well the potential for exploiting SWI/SNF complex abrogation therapeutically are important future areas of renal cancer research.

\section{Methods Summary}

DNA samples from ccRCC patients tumour and matching normal were all obtained under local IRB and LREC approvals for this study and processed as previously described ${ }^{2}$. DNA fragmentation, library preparation and solution phase hybrid capture were according to manufacturer instructions (Agilent Technologies, US) and modified from previously published protocols ${ }^{5}$. Capillary-based Sanger sequencing for confirmations and PBRM1 followup were done as previously described ${ }^{2}$ with manual inspection of all sequencing traces. mRNA was extracted from snap-frozen mouse pancreatic lesions and subjected to RT-PCR using a nested PCR approach utilising primers of mouse Pbrm1 exon 23/24 and the Carp- $\beta$-Actin Splice acceptor sequence of the T2Onc transposon cassette. Resulting bands were gel-purified and subjected to capillary-based Sanger sequencing. PBRM1 or scrambled control siRNAs (Santa Cruz, CA) were transfected into ccRCC cell lines using Lipofectamine 2000 (Invitrogen, CA) according to the manufacturer's conditions. Real-time PCR and western blotting were all done utilising standard protocols essentially as described $^{1}$. Expression analyses were carried out as previously described ${ }^{2}$.

\section{Supplementary Material}

Refer to Web version on PubMed Central for supplementary material.

\section{Acknowledgments}

PAF and MRS would like to acknowledge the Wellcome Trust for support under grant reference 077012/Z/05/Z and Allyson Coffey, Dan Turner and Lyra Mamanova for assistance with the exon capture. KF, KD and BTT acknowledge the support of the Van Andel Research Institute. BTT would like to acknowledge support from the Lee Foundation. IV is supported by a fellowship from The International Human Frontier Science Program Organization. DJA acknowledges the support of Cancer Research UK. DT and PP-M acknowledge the support of the University of Cambridge, Cancer Research UK and Hutchison Whampo and thank Dr. Will Howatt, Mr. Allen Hazelhurst and colleagues in the CRI core facilities for their support. BTT would like to dedicate this work to Tat Hock Teh.

\section{References}

1. van Haaften G, et al. Somatic mutations of the histone H3K27 demethylase gene UTX in human cancer. Nat Genet. 2009; 41:521-3. [PubMed: 19330029]

2. Dalgliesh GL, et al. Systematic sequencing of renal carcinoma reveals inactivation of histone modifying genes. Nature. 2010; 463:360-363. [PubMed: 20054297]

3. Kouzarides T. Chromatin Modifications and Their Function. Cell. 2007; 128:693-705. [PubMed: 17320507]

4. Thompson M. Polybromo-1: The chromatin targeting subunit of the PBAF complex. Biochimie. 2009; 91:309-319. [PubMed: 19084573]

5. Gnirke A, et al. Solution hybrid selection with ultra-long oligonucleotides for massively parallel targeted sequencing. Nat Biotech. 2009; 27:182-189.

6. Ye K, Schulz MH, Long Q, Apweiler R, Ning Z. Pindel: a pattern growth approach to detect break points of large deletions and medium sized insertions from paired-end short reads. Bioinformatics. 2009; 25:2865-2871. [PubMed: 19561018]

7. Reisman D, Glaros S, Thompson EA. The SWI/SNF complex and cancer. Oncogene. 2009; 28:1653-1668. [PubMed: 19234488]

8. Schneppenheim R, et al. Germline Nonsense Mutation and Somatic Inactivation of SMARCA4/ BRG1 in a Family with Rhabdoid Tumor Predisposition Syndrome. The American Journal of Human Genetics. 2010; 86:279-284. 
9. Versteege I, et al. Truncating mutations of hSNF5/INI1 in aggressive paediatric cancer. Nature. 1998; 394:203-206. [PubMed: 9671307]

10. Wong AKC, et al. BRG1, a Component of the SWI-SNF Complex, Is Mutated in Multiple Human Tumor Cell Lines. Cancer Res. 2000; 60:6171-6177. [PubMed: 11085541]

11. Chandrasekaran R, Thompson M. Polybromo-1-bromodomains bind histone H3 at specific acetyllysine positions. Biochemical and Biophysical Research Communications. 2007; 355:661-666. [PubMed: 17320048]

12. Xia W, et al. BAF180 Is a Critical Regulator of p21 Induction and a Tumor Suppressor Mutated in Breast Cancer. Cancer Research. 2008; 68:1667-1674. [PubMed: 18339845]

13. Sekine I, et al. The 3 p21 candidate tumor suppressor gene BAF180 is normally expressed in human lung cancer. 2005; 24:2735-2738.

14. Jones S, et al. Core Signaling Pathways in Human Pancreatic Cancers Revealed by Global Genomic Analyses. Science. 2008; 321:1801-1806. [PubMed: 18772397]

15. Keng VW, et al. A conditional transposon-based insertional mutagenesis screen for genes associated with mouse hepatocellular carcinoma. Nat Biotech. 2009; 27:264-274.

16. Hingorani SR, et al. Preinvasive and invasive ductal pancreatic cancer and its early detection in the mouse. Cancer Cell. 2003; 4:437-450. [PubMed: 14706336]

17. Starr TK, et al. A transposon-based genetic screen in mice identifies genes altered in colorectal cancer. Science. 2009; 323:1747-50. [PubMed: 19251594]

18. Burrows AE, Smogorzewska A, Elledge SJ. Polybromo-associated BRG1-associated factor components BRD7 and BAF180 are critical regulators of $\mathrm{p} 53$ required for induction of replicative senescence. Proc Natl Acad Sci U S A. 2010; 107:14280-14285. [PubMed: 20660729]

19. Xue Y, et al. The human SWI/SNF-B chromatin-remodeling complex is related to yeast Rsc and localizes at kinetochores of mitotic chromosomes. Proc Natl Acad Sci U S A. 2000; 97:1301513020. [PubMed: 11078522]

20. Vries RGJ, et al. Cancer-associated mutations in chromatin remodeler hSNF5 promote chromosomal instability by compromising the mitotic checkpoint. Genes Dev. 2005; 19:665-670. [PubMed: 15769941]

21. Mandriota SJ, et al. HIF activation identifies early lesions in VHL kidneys: Evidence for sitespecific tumor suppressor function in the nephron. Cancer Cell. 2002; 1:459-468. [PubMed: 12124175]

22. Young AP, et al. VHL loss actuates a HIF-independent senescence programme mediated by $\mathrm{Rb}$ and p400. Nat Cell Biol. 2008; 10:361-369. [PubMed: 18297059]

23. Steven CC, Amanda HP, Nabeel AA, Charles HCMB, Eamonn RM. Inactivation of the von Hippel-Lindau $(V H L)$ tumour suppressor gene and allelic losses at chromosome arm 3p in primary renal cell carcinoma: Evidence for a $V H L$-independent pathway in clear cell renal tumourigenesis. Genes, Chromosomes and Cancer. 1998; 22:200-209. [PubMed: 9624531]

24. Kenneth NS, Mudie S, van Uden P, Rocha S. SWI/SNF Regulates the Cellular Response to Hypoxia. Journal of Biological Chemistry. 2009; 284:4123-4131. [PubMed: 19097995]

25. Wang X, et al. Expression of p270 (ARID1A), a component of human SWI/SNF complexes, in human tumors. Int J Cancer. 2004; 112:636-642. [PubMed: 15382044]

26. Jones S, et al. Frequent Mutations of Chromatin Remodeling Gene ARID1A in Ovarian Clear Cell Carcinoma. Science. 2010 [PubMed: 20826764]

27. Wiegand KC, et al. ARID1A Mutations in Endometriosis-Associated Ovarian Carcinomas. N Engl J Med. 2010

28. Papaemmanuil E, et al. Loci on 7p12.2, 10q21.2 and 14q11.2 are associated with risk of childhood acute lymphoblastic leukemia. Nat Genet. 2009; 41:1006-1010. [PubMed: 19684604]

29. Collier LS, Carlson CM, Ravimohan S, Dupuy AJ, Largaespada DA. Cancer gene discovery in solid tumours using transposon-based somatic mutagenesis in the mouse. Nature. 2005; 436:272276. [PubMed: 16015333]

30. Uren AG, et al. Large-Scale Mutagenesis in p19ARF- and p53-Deficient Mice Identifies Cancer Genes and Their Collaborative Networks. Cell. 2008; 133:727-741. [PubMed: 18485879] 


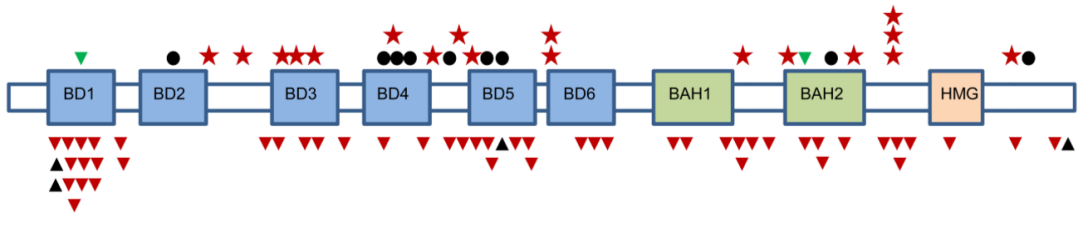

Figure 1. PBRM1 somatic mutations

Representation of PBRM1 transcript with boxes BR1-BR6, BAH1-2 and HMG indicating the positions of the bromodomains 1-6, bromo-adjacent homology domains and highmobility group domain, respectively. Relative positions of mutations are indicated by symbols. Stars - nonsense, dots - missense, red triangles - frameshift deletions, black triangles - frameshift insertions and green triangles - in-frame deletions. Splice-site mutations are not depicted. 




Figure 2. Analysis of PBRM1 missense mutations

Bars represent histogram of the mean score of in silico generated random missense mutations (10,000 sets of three mutations that can be scored) and the red circle denotes the mean score of the somatic mutations that could be scored (T232P $\square \mathrm{s}=-7.78$, A597D $\square \mathrm{s}=$ $-9.69, \mathrm{H} 1204 \mathrm{P} \square \mathrm{s}=-2.76$ ). The somatic set is significantly different from the null set (pvalue 0.01 ). They have a higher negative mean score and are thus predicted to be more deleterious on average. 




Figure 3. Pbrm1 is frequently mutated in a mouse model of pancreatic cancer To identify genes that co-operate with $K$-Ras in the formation of pancreatic cancer a conditional allele of $K-\operatorname{Ras}^{G 12 D}$ and $P d x 1$-Cre were combined with a conditional Sleeping Beauty transposase driver and the T2Onctg transposon donor allele ${ }^{29}$. Expression of Cre results in expression of $K-\operatorname{Ras}^{G 12 D}$ and transposon mobilization within the epithelial compartment of the pancreas. Isolation of the transposon insertion sites from a panel of 153 pancreatic cancers and pre-neoplastic lesions generated from this model revealed a common insertion site in Pbrm1 suggesting that loss of Pbrm 1 co-operates with $K$-Ras $G 12 D$ in pancreatic cancer development. Statistical analysis was performed as previously described $^{30}$. Transposon insertions in the forward strand of Pbrm1 are shown in green. Insertions in the reverse orientation are shown in red. A chromatogram from sequencing of RT-PCR products from one tumour is shown demonstrating splicing of exon 24 of Pbrm 1 into the inserted transposon, thus truncating the transcript. 
A





Day 0 Day 1 Day 2 Day 3

C 30



D









Figure 4. Knockdown of PBRM1 expression in RCC cell lines

(A) Verification of PBRM1 knockdown by western blotting. (B)Silencing PBRM1 increased the proliferation of ACHN and 786-O with wild type PBRM1, but not A704 with a homozygous $P B R M 1$ truncating mutation. Data represent means of triplicate experiments with standard deviation, $p<0.01$. (C) Knockdown of $P B R M 1$ enhanced colony formation in SN12C cells. Data represent means of triplicate experiments with standard deviation, $p<0.01$. (D) Knockdown of $P B R M 1$ enhanced cell migration in 786-O, SN12C and TK10 cells. Data represent means of triplicate experiments with standard deviation, $p<0.01$. (E) Gene sets that are most significantly deregulated following PBRM1 knockdown in three RCC cell lines using curated gene sets obtained from MSigDB (http:// www.broadinstitute.org/gsea/msigdb/) and additional curated gene sets obtained from the PGSEA package (see Supplemental Material for details). 